\title{
Development of a pharmacogenetic- based warfarin dosing algorithm and its performance in Brazilian patients: highlighting the importance of population-specific calibration
}

\begin{abstract}
Background: The main aims of the present study were to develop a pharmacogeneticbased warfarin dosing algorithm and to validate it in a highly admixed population. Materials \& methods: We included two patient cohorts treated with warfarin (first cohort, $\mathrm{n}=832$; and second cohort, $\mathrm{n}=133$ ). Results: Our algorithm achieved a determination coefficient of $40 \%$ including the variables age, gender, weight, height, self-declared race, amiodarone use, enzyme inducers use, VKORC1 genotypes and predicted phenotypes according to CYP2C9 polymorphisms. Conclusion: Data suggest that our developed algorithm is more accurate than the IWPC algorithm when the application is focused on patients from the Brazilian population. Population-specific derivation and/or calibration of warfarin dosing algorithms may lead to improved performance compared with general use dosing algorithms currently available.
\end{abstract}

Original submitted 26 November 2014; Revision submitted 9 April 2015

Keywords: algorithm $\bullet C Y P 2 C 9 \bullet V K O R C \bullet$ warfarin

Warfarin is the most widely prescribed oral anticoagulant agent worldwide [1]. This drug is used in antithrombotic therapy and prevention of thrombosis [2]. Stable maintenance warfarin dose is difficult to establish due to a narrow therapeutic window and great inter-individual variability on its pharmacodynamics and pharmacokinetics responses. As such, warfarin therapy must be monitored by the international normalized ratio (INR). Indeed, several studies showed that the highest the INR the higher is the increase in the risk of bleeding, whereas low INRs are associated with increased risk of thromboembolism or stroke [3,4].

Furthermore, studies showed the association between warfarin dose and polymorphisms in the gene encoding the cytochrome P-450 2C9 isoenzyme (CYP2C9) and the vitamin $\mathrm{K}$ epoxide reductase complex 1 (VKORC1). CYP2C9 isoenzyme is responsible for metabolizing the active $S$-warfarin enantiomer and VKORC1 recycles reduced vitamin $\mathrm{K}$ during the activation process of the clotting factors [5-7]. In 2007, the US FDA added information to the warfarin product label, highlighting the importance of the pharmacogenetic effect on the product, especially regarding $C Y P 2 C 9^{*} 2, C Y P 2 C 9^{*} 3$ and $V K O R C 11639 \mathrm{G}>\mathrm{A}$ polymorphisms. In 2011, the FDA reviewed the information of pharmacogenetic effect on maintenance warfarin doses based on CYP2C9 and VKORC1 genotypes for the clinicians [8-10].

Since then, pharmacogenetic algorithms for predicting warfarin dose have been proposed, taking advantage of clinical, concurrent medication and genetic data. Nonetheless, there is still dispute whether warfarin therapy guided by genotype information improves this pharmacotherapy and what is the cost-benefit of this approach [11]. Pirmohamed et al. followed up, for 12 weeks, patients who were recruited in the UK and Sweden. They identified that the mean percentage of time in the therapeutic range (TTR) was higher in the genotype-guided group $(67.4 \%)$ compared with the control
Paulo Caleb Junior Lima Santos ${ }^{*, 1}$, Leiliane Rodrigues Marcatto', Nubia Esteban Duarte', Renata Alonso Gadi Soares', Celia Maria Cassaro Strunz $z^{2}$ Maurício Scanavacca ${ }^{3}$, Jose Eduardo Krieger ${ }^{1}$ \& Alexandre Costa Pereira $^{1}$

'Laboratory of Genetics \& Molecular Cardiology, Heart Institute (InCor), University of São Paulo Medical School, Av. Dr. Eneas de Carvalho Aguiar, 44 Cerqueira Cesar, São Paulo, CEP 05403-000, Brazil

${ }^{2}$ Clinical Laboratory, Heart Institute (InCor), University of São Paulo Medical School, São Paulo, Brazil ${ }^{3}$ Clinical Cardiology Division, Heart Institute (InCor), University of São Paulo Medical School, São Paulo, Brazil *Author for correspondence: Tel.: +55 1126615929 Fax: +55 1126615022 pacaleb@usp.br 
group $(60.3 \%, \mathrm{p}<0.001)$ [12]. Verhoef et al. studied patients taking acenocoumarol or phenprocoumon recruited in The Netherlands and Greece. They identified that genotype-guided dosing did not improve the TTR during the first 12 weeks after the initiation of therapy (61.6 and 60.2\%; p = 0.52). However, they identified different TTR during the first 4 weeks after the initiation of treatment in the pharmacogenetic and control groups (52.8 and 47.5\%; p = 0.02) [13]. Kimmel et al., studying patients from 18 clinical centers in the USA, concluded that genotype-guided dosing of warfarin did not improve anticoagulation control during the first 4 weeks of therapy [14]. Likely, the different findings observed in these three published randomized, controlled trials [12-14] might be, at least partially, related to different sampled populations and study designs, including slightly different dosing-algorithms.

In this scenario, the main aims of this study were to develop a population-specific pharmacogeneticbased warfarin dosing algorithm and to compare the performance of the newly derived dosing-scheme with internationally available algorithms.

\section{Materials \& methods}

\section{Patients \& study design}

This is a cross-sectional study that included two patient cohorts treated with warfarin for at least 12 months, prior to enrollment, at the Heart Institute (Incor), University of São Paulo, São Paulo, Brazil. They were enrolled in two different time periods: from September, 2011 to March, 2012 (first cohort, $\mathrm{n}=832$ ) and from January 2014 to February 2014 (second cohort, $\mathrm{n}=133$ ). The study protocol was approved by the Institutional Ethics Committee (Register Number 0804/10), and written informed consent was obtained from all participants prior to entering the study. Patients with chronic liver failure, using other anticoagulant drugs, receiving chemotherapy and who referred alcoholism were excluded. Patients were separated in self-declared 'racial/color' subgroups according to the criteria used by the Brazilian Census, as White, Intermediate (meaning Brown, Pardo in Portuguese) or Black $[15,16]$. Data regarding current drug use were obtained through a standardized interview with a pharmacist and checked on electronic medical records.

\section{Derivation, replication \& performance testing} phases of the pharmacogenetic-based warfarin dosing algorithm

Figure 1 shows the analytical design used.

Warfarin doses were determined by the physicians who were unaware of the CYP2C9 and VKORC1 genotypes. HAS-BLED score was used to estimate the risk of bleeding on anticoagulation therapy. Patients who did not have increased risk were prescribed $5 \mathrm{mg} /$ day of warfarin $(35 \mathrm{mg} /$ week), while patients who had increased risk were prescribed $2.5 \mathrm{mg} /$ day of warfarin (17.5 mg/week) [17].

Algorithm derivation was made using data from patients from the first cohort that, at enrollment, presented on warfarin stable maintenance dose $(n=368)$, in other words, achievement of the therapeutic target defined as three consecutive values of INR between 2.0 and 3.0 (including the current test). Patients with INR values from 1.8 to $<2.0$ or from $>3.0$ to $3.2 \mathrm{did}$ not change their warfarin doses; however, INR was repeated in 7 days.

Dose-prediction model was derived in the following way (derivation sample, $\mathrm{n}=368$ ). First, we adjusted multiple linear regression models to select the variables to be included in our algorithm. Initial selection was based on the already existing IWPC warfarin-dosing algorithm. The model was selected according to the coefficient of determination $\mathrm{R}^{2}$ and the significance of each covariate added to the model. Next, we performed a series of permutations to define the best and more stable weighting scheme for each covariate. This was performed by dividing the sample into two random subgroups (276 and 92) and, in each subgroup, adjusting the preselected multiple linear regression model. The effects of the covariates of interest on warfarin maintenance dose, as well as, the goodness of fit of the model by $\mathrm{R}^{2}$ were saved for the two generated models (i.e., using 276 and 92 individuals). In order to verify the stability of the effects and adjustment, this process was repeated 10,000-times (details in the 'Results' section). Finally, the average effect of each covariate on the 10,000 permutations was obtained and these values are proposed for the final algorithm.

A sample of 133 patients in current stable dose was enrolled in a different time period to be used as a replication sample of the derived algorithm (second cohort).

In a performance testing phase, a sample was then formed from patients who were not on stable dose at the enrollment period of the first cohort $(n=464)$. Furthermore, a follow-up evaluation was made with patients on stable dose from the first cohort $(n=368)$. They were followed up for 1 year $(\mathrm{n}=318)$, individual TTRs were calculated and patients were divided into two groups according to the median TTR $(<0.73$ or $\geq 0.73)$ or with the presence of all INR values within the therapeutic target during the one-year follow-up period. This analysis was used as a proxy of anticoagulation stability.

\section{Measures \& outcomes}

Oral anticoagulant therapy was assessed by the prothrombin time (PT) using an automated coagulometric method. INR calculation was obtained by the 


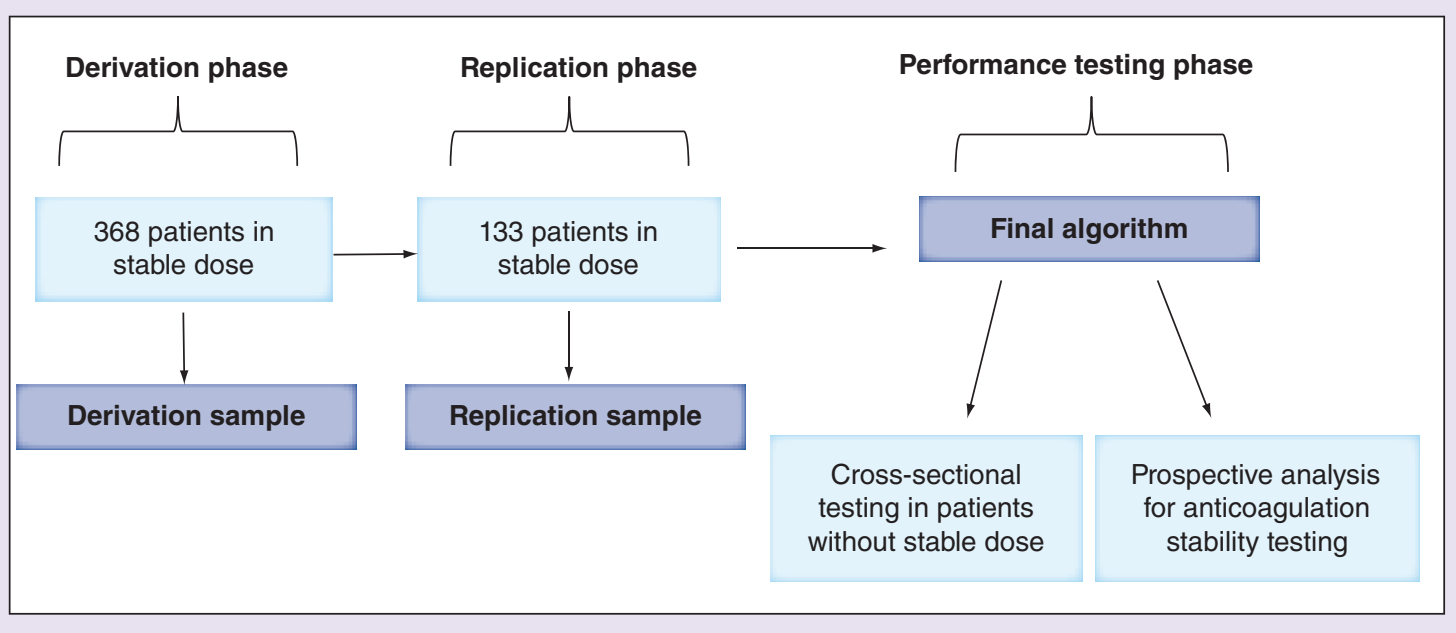

Figure 1. Analytical design: derivation, replication and performance testing phases.

ratio PT of the patient/normal PT controls, elevated to the international sensitivity index. Venous blood samples were collected in tubes containing sodium citrate $3.8 \%$. Follow-up of INR for dose adjustment was decided according to physician discretion based on guideline recommendations $[2,18]$.

Blood samples were collected to assess current INR and DNA analysis. Besides current INR, past INR values were checked in electronic medical records from the 12 months preceding enrollment. TTR was calculated by the Rosendaal method [19], which uses linear interpolation to assign an INR value to each day between successive observed INR values.

Major bleeding was defined mainly as fatal bleeding, intrapericardial bleeding with cardiac tamponade, intracranial bleeding, severe hypotension or hypovolemic shock due to bleeding and requiring either vasopressors or surgical intervention, a decline in hemoglobin of $5.0 \mathrm{~g} / \mathrm{dL}$ or more after adjusting for red blood cell transfusions. Minor bleeding was classified as any bleeding event requiring medical intervention but not meeting the criteria for major bleeding [20].

\section{Genotyping \& predicted metabolic phenotypes} according to CYP2C9 \& VKORC1 polymorphisms Genomic DNA was extracted from peripheral blood leukocytes by the standard salting-out method. Genotyping of CYP2C9*2 (c.430C > T, rs1799853), CYP2C9*3 (c.1075A > C, rs1057910) and VKORC1 3673 (g.1639G > A, rs9923231) polymorphisms was detected by polymerase chain reaction followed by restriction enzyme digestion [21,22]. As quality control, $6 \%$ of the samples were reanalyzed and gave identical results.

Patients were divided into distinct predicted phenotypes: extensive metabolizer (EM: wild-type genotypes for the CYP2C9 polymorphisms $\left.-{ }^{*} 1 /{ }^{*} 1\right)$, inter- mediate metabolizer (IM: heterozygous genotypes for the loss-of-function CYP2C9 polymorphisms - ${ }^{*} 1 /{ }^{*} 2$ or ${ }^{*} 1 /{ }^{*} 3$ ) and poor metabolizer (PM: mutant homozygous or compound heterozygous genotypes for the loss-of-function CYP2C9 polymorphisms - ${ }^{*} 2 /{ }^{*} 2$ or $* 3 / * 3$ or $\left.{ }^{*} 2 / * 3\right)$ [23].

\section{Statistical analysis}

Categorical variables are presented as percentages, while continuous variables are presented as mean \pm standard deviation or standard error of the mean. Chi-square test was performed for the comparative analysis of the categorical variables (such as adverse events, and Hardy-Weinberg equilibrium calculation) according to the polymorphisms status. The Student's $t$-test was used for comparing differences between predicted doses (PD), observed doses (OD) and 'difference in weekly dose' between OD and PD; and for comparing warfarin dose, and TTR according to predicted metabolic phenotypes due to CYP2C9 polymorphisms. One-way ANOVA (analysis of variance) test was used for the analysis of the warfarin dose and TTR according to VKORC1 genotypes. Tukey's post hoc test was performed to identify the different groups. Mean warfarin dose was adjusted for age, gender, BMI and race. Multiple logistic regression analysis was used to identify variables associated with minor or major bleedings. Pearson correlation was performed to evaluate the correlation between observed and predicted doses. In the multiple linear regression models the following variables were included: age (years), gender, weight $(\mathrm{kg})$, height $(\mathrm{cm})$, self-declared race, amiodarone use, enzyme inducers use, VKORC1 genotypes and predicted phenotypes due to CYP2C9 polymorphisms (EM, IM or PM). The level of significance set at $\mathrm{p} \leq 0.05$. All statistical analyses were car- 
ried out using SPSS software (v. 16.0) and R project for statistical computing (r-project.org/).

\section{Results}

Demographic \& clinical characteristics

Table 1 shows demographic and clinical characteristics of the patients from the first cohort. Of the 832 patients (mean age $63 \pm 14$ ), 417 (50.1\%) were female. Main indications for treatment with warfarin therapy were atrial fibrillation or flutter, previous cerebrovascular accident, thrombosis or embolus and use of prosthetic heart valves (excluding mechanical valves) requiring chronic anticoagulation (Table 1).

Patients on stable maintenance dose formed the derivation sample $(\mathrm{n}=368,44.2 \%)$. All patients took warfarin for at least 1 year prior to enrollment and the average time of anticoagulation of the study patient group was $6.1 \pm 5.2$ years.

\section{Frequencies of VKORC1 \& CYP2C9}

polymorphisms \& predicted metabolic phenotypes

Table 1 summarizes frequencies of genotypes and predicted metabolic phenotype of the patients from the first and second cohorts. The frequencies of the A variant allele and of the homozygous genotype for the VKORC1 g.1639G > A were 32.5 and $12.3 \%$, respectively. The frequencies of the $C Y P 2 C 9^{*} 2$ and $C Y P 2 C 9^{*} 3$ variant alleles were 10.8 and $4.6 \%$, respectively. The frequencies of the predicted metabolic phenotypes were: $73.4 \%$ for EM, $22.5 \%$ for IM and $4.1 \%$ for PM. The genotypic distributions for the VKORC1 g.1639G > A and CYP2C9 polymorphisms were not in accordance with the Hardy-Weinberg equilibrium, probably because of the significant genetic structure at this locus in the Brazilian population, as previously reported by our group [15].

Table 1. Demographic, clinical and genetic characteristics of the study patients from first and second cohorts.

\begin{tabular}{|c|c|c|c|}
\hline Variables & $\begin{array}{l}\text { First cohort } \\
n=832(100 \%)\end{array}$ & $\begin{array}{l}\text { Second cohort } \\
n=133(100 \%)\end{array}$ & p-value \\
\hline Gender, female (\%) & 50.1 & 43.6 & 0.20 \\
\hline Age (years) & $63 \pm 14$ & $63 \pm 17$ & 0.67 \\
\hline BMI $\left(\mathrm{kg} / \mathrm{m}^{2}\right)$ & $27 \pm 5$ & $26 \pm 4$ & 0.80 \\
\hline \multicolumn{4}{|l|}{ Race/color (\%): } \\
\hline - White & 68.7 & 56.4 & 0.01 \\
\hline - Brown & 20.6 & 23.3 & \\
\hline - Black & 8.1 & 17.3 & \\
\hline - Other ${ }^{\dagger}$ & 2.6 & 3.0 & \\
\hline Smoking (\%) & 5.7 & 3.0 & \\
\hline \multicolumn{4}{|l|}{ Indication for treatment (\%) } \\
\hline Atrial fibrillation or flutter & 59.3 & 63.2 & 0.39 \\
\hline Cerebrovascular accident & 12.8 & 9.0 & \\
\hline Thrombosis or embolus & 6.3 & 6.0 & \\
\hline Prosthetic heart valve & 13.5 & 15.8 & \\
\hline Other & 8.1 & 6.0 & \\
\hline \multicolumn{4}{|l|}{ VKORC1 1639 G > A (\%) } \\
\hline GG & 47.2 & 38.4 & 0.06 \\
\hline GA & 40.5 & 54.1 & \\
\hline$A A$ & 12.3 & 7.5 & \\
\hline \multicolumn{4}{|c|}{ 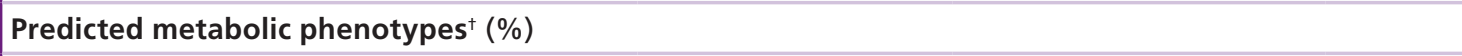 } \\
\hline $\mathrm{EM}(* 1 / * 1)$ & 73.4 & 75.2 & 0.68 \\
\hline $\operatorname{IM~}(* 1 / * 2$ or $* 1 / * 3)$ & 22.5 & 21.8 & \\
\hline $\operatorname{PM}(* 2 / * 2, * 3 / * 3$ or $* 2 / * 3)$ & 4.1 & 3.0 & \\
\hline
\end{tabular}


Mean warfarin dose, adverse events \& frequencies of patients within therapeutic range according to VKORC1 \& CYP2C9 polymorphisms

In the overall group from the first cohort $(n=832)$, mean warfarin dose was significantly lower in patients carrying IM+PM predicted phenotypes $(25.8 \pm 0.8 \mathrm{mg} /$ week $)$ compared with EM patients $(29.7 \pm 0.5 \mathrm{mg} /$ week $)(\mathrm{p}<0.001)$. For the VKORC1 polymorphism, patients carrying GG genotype had higher mean warfarin dose $(32.3 \pm 0.6 \mathrm{mg} /$ week $)$ compared with patients carrying AA genotype $(21.5 \pm 1.2 \mathrm{mg} /$ week $)(p<0.001)$. Figure 2 shows that warfarin dose was also significantly different according to polymorphisms in the patients from the derivation sample $(\mathrm{n}=368)$, even after adjustment for covariates.

TTR values and frequency of patients on stable dose were not different between EM patients $(61.4 \pm 24.5 \%$ and $44.8 \%$, respectively) and IM + PM patients $(62.0 \pm 23.7 \%$ and $44.0 \%$, respectively) $(\mathrm{p}=0.78$ and $\mathrm{p}=0.84$, respectively). Also for $V K O R C 1$ genotypes, the TTR and the frequency of patients within the therapeutic target were not different.

Regarding adverse events, frequency of a previous history of major bleedings was not different according to genotypes. However, frequency of a previous history of minor bleedings was more frequent in IM + PM patients (55.7\%) compared with EM patients
$(45.0 \% ; \mathrm{p}=0.007)$. A tendency was observed for a lower frequency of minor bleedings in patients carrying GG genotype for the VKORC1 polymorphism (GG: $43.5 \%$, GA: $52.2 \%$, AA: $50.0 \%$; p = 0.06). In a multivariate logistic regression analysis, the IM + PM was a variable associated with minor bleedings (OR: $1.49,95 \%$ CI: $1.07-2.08 ; \mathrm{p}=0.02$ ), in a model including the covariates age, gender, race, BMI, amiodarone use, enzyme inducers use and VKORC1 genotypes.

\section{Derivation of the pharmacogenetic-based warfarin dosing algorithm}

Table 2 shows the final model for the developed algorithm. The model was developed with 368 patients in stable dose (derivation sample). The construction of the model achieved a multiple $\mathrm{R}^{2}$ of $40 \%$ including nine variables.

The inclusion of the variables such as statin use, proton-pump inhibitors use, indication for treatment and smoking did not improve the algorithm performance, as well as, they did not present significant $\mathrm{p}$ values on multiple or simple linear regression models. Carbamazepine and phenytoin were the CYP enzyme inducers considered in this analysis. Supplementary Figure 1 (see online at: www.futuremedicine.com/doi/suppl/10.2217/pgs.15.48) shows histograms of distributions of the $\mathrm{R}^{2}$ for the two

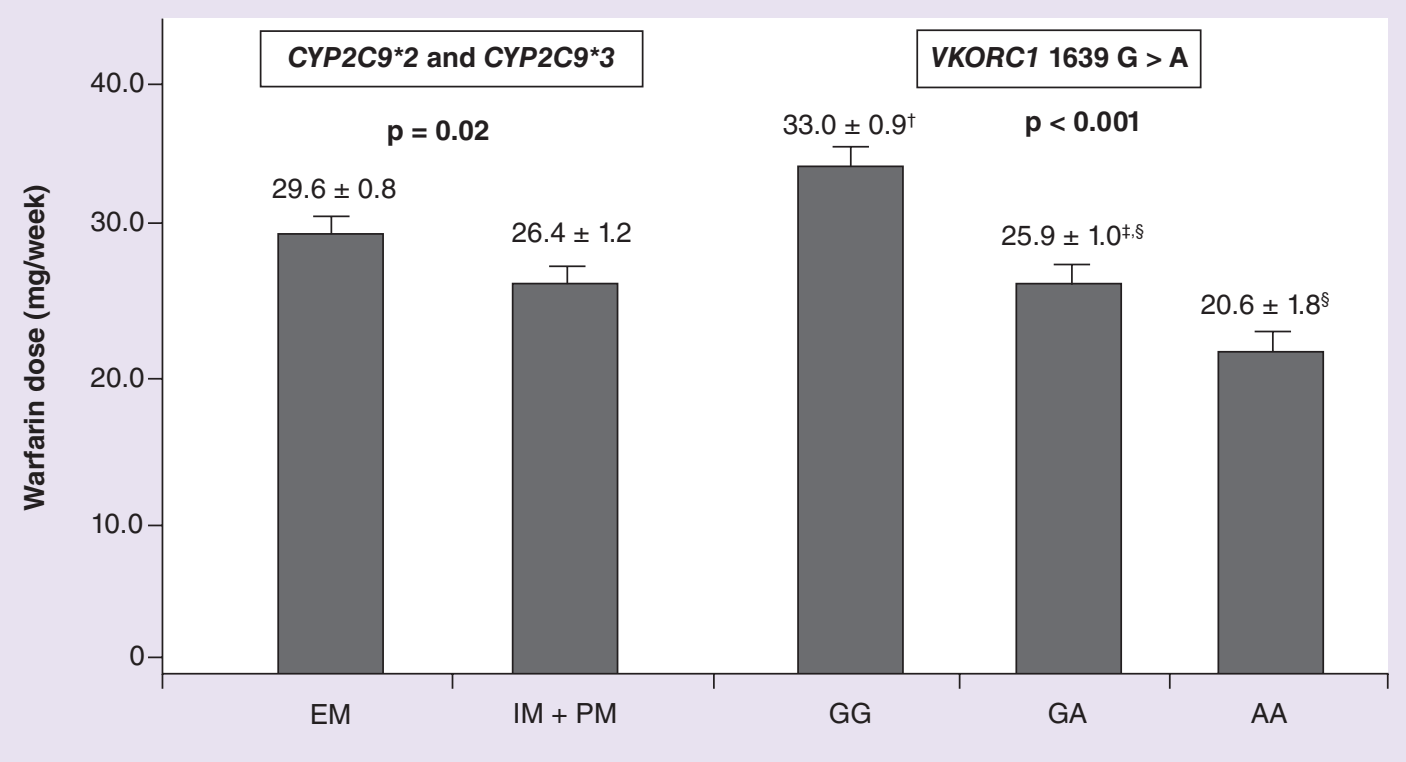

Figure 2. Warfarin dose in the patients on stable maintenance dose (derivation sample) according to CYP2C9 and VKORC1 polymorphisms from first cohort $(n=368)$. CYP2C9*2 c.C445T ( $r$ 1799853), CYP2C9*3 c.A1100C (rs1057910) and VKORC1 c.G1639A (rs9923231) polymorphisms. Data are expressed as mean \pm standard error. Values for the VKORC1 genotypes (GG, $n=174 ; G A, n=151 ; A A, n=43$ ) with different superscript symbols $\left({ }^{+1, t, \S}\right)$ are significantly different (Tukey's post hoc test). These data were adjusted for age, gender, BMI and race. Stable maintenance dose means three consecutive values of INR between 1.8 and 3.2 (including the current test). EM: Extensive metabolizer $(n=269)$; IM: Intermediate metabolizer; PM: Poor metabolizer (IM + PM, $n=99)$. 
Table 2. Variables of the warfarin dosing algorithm in a multiple linear regression model.

\begin{tabular}{|c|c|c|}
\hline Variable & $\begin{array}{l}\beta \text { coefficient } \\
\text { (standard deviation) }\end{array}$ & $95 \% \mathrm{Cl}$ \\
\hline Age & $-0.16(0.03)$ & $-0.16,-0.15$ \\
\hline Gender & $-1.65(0.80)$ & $-1.66,-1.63$ \\
\hline Weight & $0.15(0.02)$ & $0.15,0.15$ \\
\hline Height & $0.08(0.06)$ & $0.07,0.08$ \\
\hline 'Race/color' & $0.36(0.61)$ & $0.34,0.37$ \\
\hline Amiodarone use & $-7.34(0.87)$ & $-7.35,-7.31$ \\
\hline Enzyme inducers use & $22.42(6.41)$ & $22.29,22.55$ \\
\hline VKORC1 genotypes & $-6.68(0.53)$ & $-6.69,-6.67$ \\
\hline CYP2C9 polymorphisms & $-2.52(0.75)$ & $-2.53,-2.50$ \\
\hline \multicolumn{3}{|c|}{$\begin{array}{l}\text { Characteristics of the variables: age (years); gender (1, if male, otherwise 0); weight (kg); } \\
\text { height (cm); 'race/color' (self-declared as White, Brown or Black - 1, } 2 \text { and 3, respectively); } \\
\text { amiodarone use (1, if patient took amiodarone, otherwise 0); enzyme inducers use (1, if } \\
\text { patient took inducers drugs, otherwise 0); VKORC1 genotypes (GG, GA or AA - 0, } 1 \text { and } \\
\text { 2, respectively); and predicted metabolic phenotypes according to CYP2C9 polymorphisms } \\
\text { (extensive metabolizer, intermediate metabolizer, poor metabolizer: - 0, } 1 \text { and 2, } \\
\text { respectively). } \\
\text { CYP2C9*2, c.430C > T (rs1799853), CYP2C9*3, c.1075A > C (rs1057910), and VKORC1 } \\
\text { g.1639G > A (rs9923231) polymorphisms. }\end{array}$} \\
\hline
\end{tabular}

groups ( $3 / 4$ and $1 / 4$ due to overall 368 from derivation sample). Also, Supplementary Figure 2 shows a histogram of distributions of the $\mathrm{R}^{2}$ variation.

In the derivation sample $(\mathrm{n}=368)$, Figure 3 shows a moderate correlation between PD and OD $\left(\mathrm{r}^{2}=0.51\right.$, $\mathrm{p}<0.001)$. In addition, Supplementary Figure 3 shows a strong correlation between PD by our algorithm and PD by IWPC algorithm $\left(\mathrm{r}^{2}=0.87, \mathrm{p}<0.001\right)$.

\section{Replication phase of the pharmacogenetic-} based warfarin dosing algorithm

Figure 4 shows the correlation between PD and OD in the replication sample $(\mathrm{n}=133)$. Interestingly, when comparing the correlation between the predicted dose by the derived algorithm and the empirical dose and the correlation between the predicted doses derived from the IWPC, Botton's and Perini's algorithms and the empirical dose, we can observe a lower dispersion of the data in the first correlation. We also found higher correlation coefficient $\left(\mathrm{r}^{2}\right)$ (0.61) compared with IWPC, Botton's and Perini's algorithms (0.51, 0.32 and 0.49 , respectively).

Performance testing phase of the algorithm Figure 5 shows the 'difference in weekly dose' between OD and PD by our and IWPC algorithms in the patients from first cohort. The 'difference in weekly dose' between OD and PD was lower using our algorithm compared with the IWPC algorithm $(0.5 \pm 0.6 \mathrm{mg} /$ week vs $-3.5 \pm 0.6 ; \mathrm{p}<0.001$, respectively), in the derivation group. We also observed sig- nificant findings in the 'difference in weekly dose' for patients with INR $>3.2$ or INR $<1.8$ (Figure 5).

Since we observed a lower dispersion of the data of OD versus PD by the derived algorithm compared with dispersion of the data of OD versus PD by the IWPC algorithm (Figure 4), we tested the stability of anticoagulation therapy by calculating the 'difference in weekly dose' in a prospective analysis. Patients in stable dose were followed up for 1 year $(\mathrm{n}=318)$ and divided into two groups according: median TTR value $(<0.73$ or $\geq 0.73)$ or with the presence of all INR values within the therapeutic target. Interestingly, Table 3 shows that the 'difference in weekly dose' between OD and PD by our algorithm was lower than the 'difference in weekly dose' between OD and PD by IWPC algorithm, especially in the patients with TTR $\geq 0.73$ and in the patients with the presence of all INR values within the therapeutic target $(\mathrm{p}<0.001)$ (Table 3$)$.

Supplementary Figure 4 shows the correlation between PD by the IWPC algorithm and PD by our algorithm in the patients without a stable dose upon ascertainment (Panel A: INR > 3.2 and Panel B: INR < 1.8). Patients without stable dose were those who did not have three consecutive values of INR between 1.8 and 3.2. However, their data only were included on the panels when their INR values were out of therapeutic range in the current test, which is paired with the current dose. The overall distribution of patients was 368 patients with stable dose, 85 patients with INR $>3.2,88$ patients with INR $<1.8$ and 291 patients with INR values out of therapeutic range in the past two tests, or one INR value $>3.2$ and another $<1.8$. Mean warfarin PD by our algorithm was lower than OD in the patients with INR $>3.2(27.2 \pm 0.8$ and $30.6 \pm 1.2 \mathrm{mg} /$ week; $\mathrm{p}=0.01$, respectively); while mean warfarin $\mathrm{PD}$ was not different than OD in the patients with INR $<1.8(27.9 \pm 0.8$ and $26.7 \pm 0.8 \mathrm{mg} /$ week; $\mathrm{p}=0.10$, respectively).

\section{Discussion}

Some studies suggested that algorithms that include genetic information, may transform the management of warfarin dosing achieving the target INR faster, safer, and in a more cost-effective manner than current practice [24-26]. However, the current cost-benefit of algorithm application in a real-life scenario is not fully understood and clinical trials have been developed to address this issue. Also, FDA released a genotyperelated dosing table, but not yet approved any dosing algorithm that will convert genotyping data into a dosing recommendation [27].

In the present study, we were able to develop and validate our algorithm in two patient cohorts. Our algorithm, composed of nine predictor variables, achieved 
a coefficient of determination $\left(\mathrm{R}^{2}\right)$ of $40.0 \%$ and was validated in a second, independent, sample from the same population. These findings suggest that our algorithm is able to adjust an optimal warfarin dose according to clinical, medication and genetic data from each patient. Some studies, including the IWPC algorithm, showed $\mathrm{R}^{2}$ values similar to ours (from 39 to $47 \%$ ) [9,24]. But, higher $\mathrm{R}^{2}$ values were observed by other studies (from 53 to 63\%) [22,25-33]. Lower observed $\mathrm{R}^{2}$ are usually associated with ethnically admixed populations and, on the other hand, higher values in homogeneous populations of European ancestry [30,34,35].

It has been suggested that the complexity of population admixture in Brazil is indeed an opportunity to explore pharmacogenomics in individuals with heterogeneous genetic ancestry under similar environmental and socioeconomical conditions as well as people who are under-represented in clinical drug trials [36]. Regarding ancestry, previous studies showed that most Brazilians share European and African ancestries, and a sizeable number of subjects also have Amerindian ancestry; with the relative ancestry proportion associated with self-declared 'color/race' and geographical regions [15,36-39].

A study with Southern Brazilian patients of European ancestry reported one of the highest $R^{2}$ values in the literature (63.3\%) [30]. The authors used a large number of variables including CYP4F2 genotype, F2 genotype, $V K O R C 1$ haplotype and several concurrent

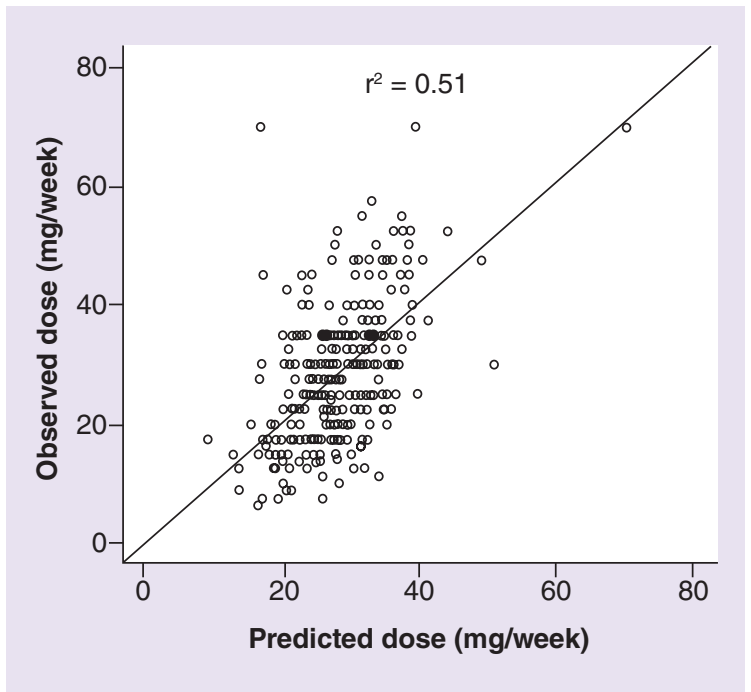

Figure 3. Correlation between predicted dose by our algorithm and observed dose $\left(n=368, r^{2}=0.51\right.$, p $<0.001$ ) in the derivation sample. Stable maintenance dose means three consecutive values of international normalized ratio between 1.8 and 3.2 (including the current test).

medications, which, taken together, have potential to add $10.6 \%$ on the model [30]. Another Brazilian study explained $51 \%$ of the warfarin dose in stable patients of mixed ancestry [31]. In our study, besides the derivation of the algorithm, we were able to perform a replication evaluation using a patient sample ascertained in a different time period. Furthermore, our algorithm was also
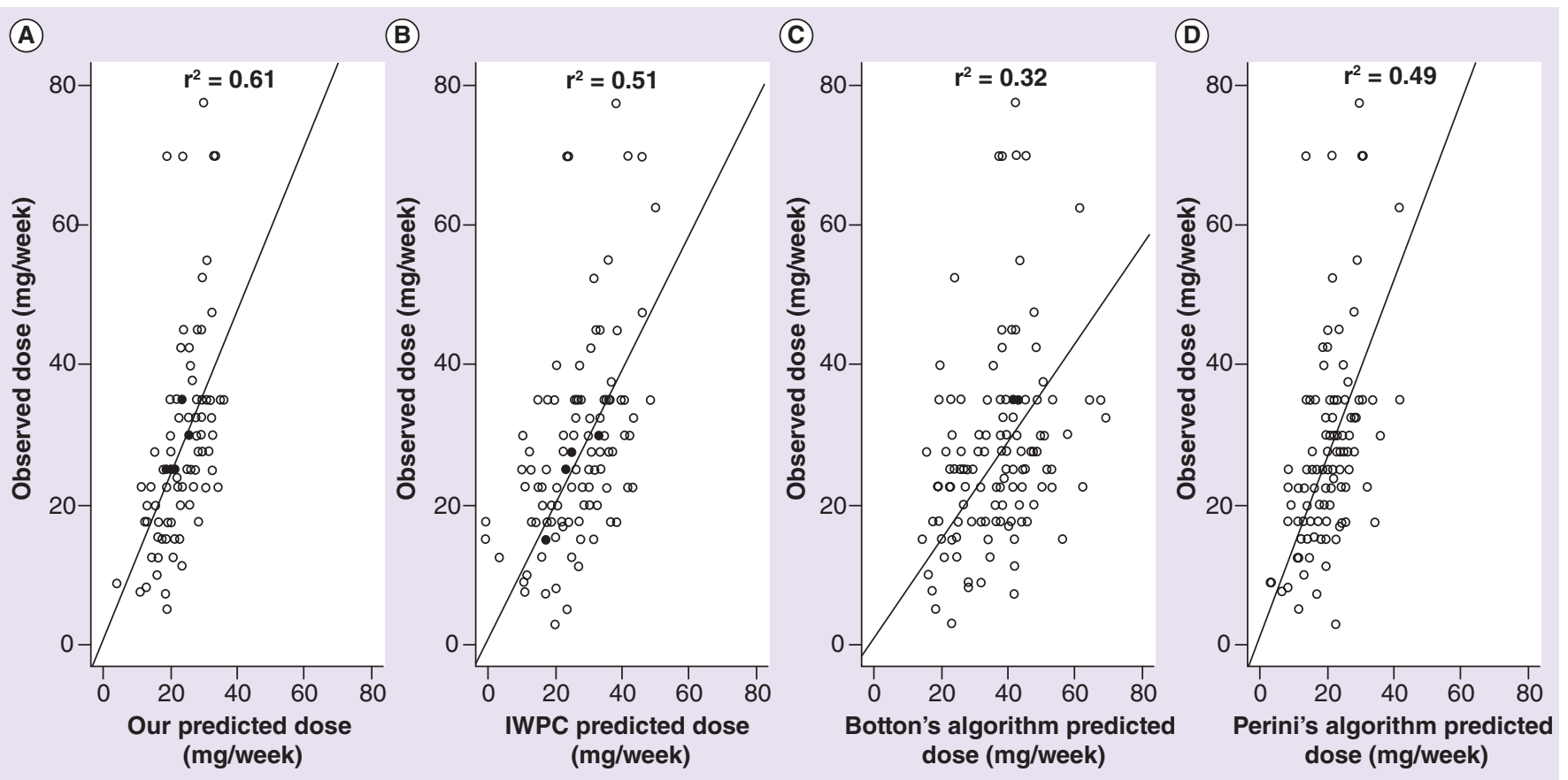

Figure 4. Correlation between predicted doses and observed dose $(n=133)$ in the validation sample. (A) Predicted dose (PD) by our algorithm $\left(r^{2}=0.61, p<0.001\right)$, (B) PD by IWPC algorithm $\left(r^{2}=0.51, p<0.001\right)$, (C) PD by Botton's algorithm $\left(r^{2}=0.32, p<0.001\right)$, and (D) PD by Perini's algorithm $\left(r^{2}=0.49, p<0.001\right)$. 

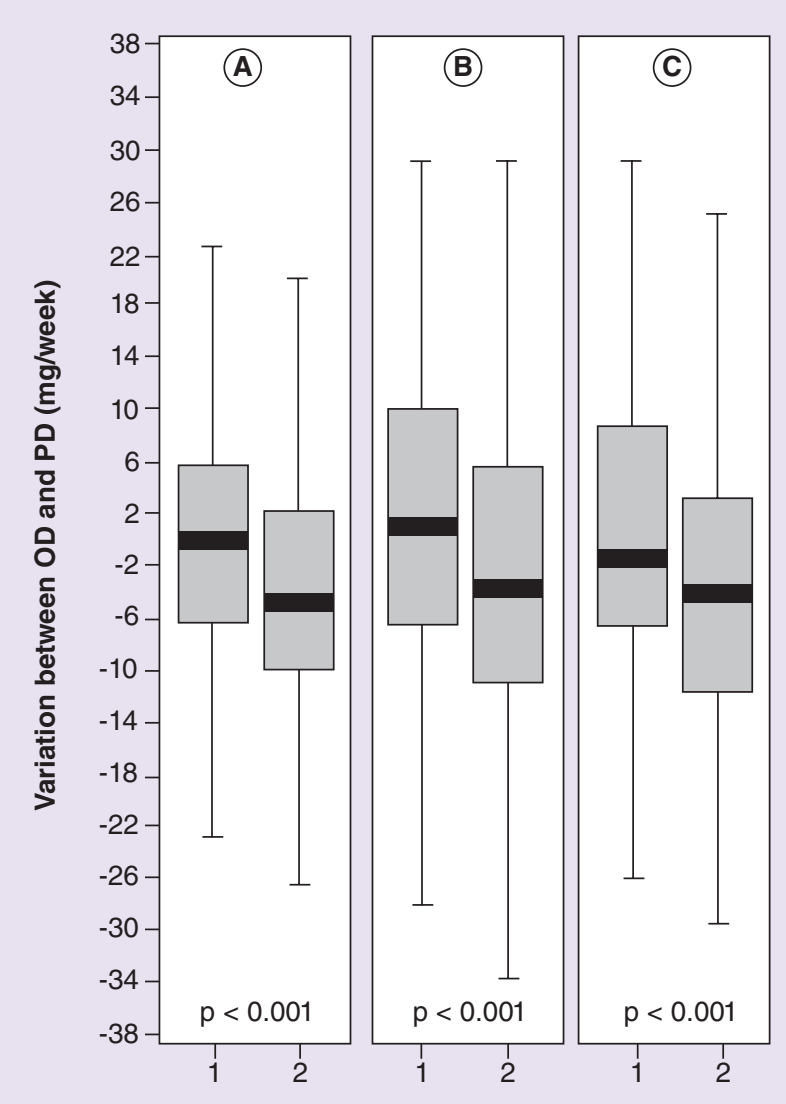

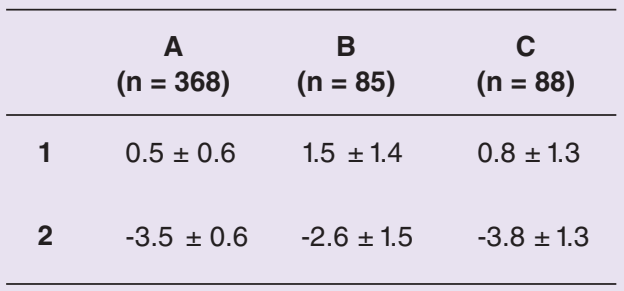

1: OD minus PD by our algorithm.

2: OD minus PD by IWPC algorithm.

Figure 5. 'Difference in weekly dose' between observed doses and predicted doses in the patients from first cohort. Group 1: PD by our algorithm. Group 2: PD by IWPC algorithm. (A) Patients with stable dose. (B) Patients with international normalized ratio $>3$.2. (C) Patients with international normalized ratio $<1.8$. 'Difference in weekly dose' was calculated subtracting PD of OD.

OD: Observed dose; PD: Predicted dose.

tested using a large number of stable patients in a followup evaluation. This analysis indicated the stability of the model and suggested that the derived algorithm applied to a Brazilian patient population of mixed ancestry to be more accurate compared with other warfarin dosing algorithms available in the literature.

Two findings of this present study suggest that our algorithm might be more accurate than the IWPC, Botton's and Perini's algorithms for individuals from the Brazilian population. First, in the replication sample, the correlation between the PD by our algorithm and the OD was higher (0.61) than the correlation between the PD by other tested algorithms and the $\mathrm{OD}(0.51,0.32$, and 0.49 , respectively). Indeed, in Figure 4 one can observe a lower dispersion of the correlation data when using the currently derived algorithm. Second, in a prospective analysis evaluating the anticoagulation stability, we observed that the 'difference in weekly dose' between OD and PD by our algorithm was lower than the 'difference in weekly dose' between OD and PD by the IWPC algorithm $(p<0.001)$. These findings suggest that the develop- ment of a population-specific algorithm might indeed capture population-specific information.

Regarding concurrent medication in this study, amiodarone and enzyme inducer drugs were significantly associated to warfarin dose and included in our algorithm; while statin use and proton-pump inhibitors use were not associated and, thus, not included. Some studies added other drugs as predictor variables in their algorithms, such as statins, amlodipine, $\beta$-blockers and diuretics, achieving an improvement of about $5 \%[26,30,31]$.

Regarding genotype variables, we also observed significant findings that are consistent with the literature. CYP2C9 and VKORC1 genotypes were associated with the warfarin stable dose and the frequencies of minor bleedings. The first association is widely known $[6,32,40,41]$. Some studies showed that CYP2C9 plus $V K O R C 1$ genotypes contribute approximately with 45 and $30 \%$ of warfarin dose interindividual variation in European and African populations, respectively [35,42-45]. Furthermore, some studies incorporated to their algorithms and other polymorphisms 
Table 3. 'Difference in weekly dose' between observed dose and predicted dose of a prospective analysis with patients on stable dose followed up for 1 year $(n=318)$.

\begin{tabular}{|c|c|c|c|c|}
\hline & \multicolumn{2}{|c|}{$\begin{array}{l}\text { Division according with TTR median } \\
\qquad(\mathrm{n}=318)\end{array}$} & \multicolumn{2}{|c|}{$\begin{array}{l}\text { Division according with the } \\
\text { presence of all INR values within } \\
\text { therapeutic target }\end{array}$} \\
\hline & $\begin{array}{l}\text { Patient group } \\
\text { with TTR }<0.73\end{array}$ & $\begin{array}{l}\text { Patient group } \\
\text { with TTR } \geq 0.73\end{array}$ & No $(n=278)$ & Yes $(n=40)$ \\
\hline OD & $29.7 \pm 1.1$ & $27.2 \pm 0.9$ & $28.6 \pm 0.8$ & $27.0 \pm 2.1$ \\
\hline OD - PD by our algorithm & $2.1 \pm 1.0$ & $-0.6 \pm 0.8$ & $1.2 \pm 0.7$ & $-2.2 \pm 1.5$ \\
\hline OD - PD by IWPC algorithm & $-2.0 \pm 1.1$ & $-4.4 \pm 0.8$ & $-2.8 \pm 0.7$ & $-5.7 \pm 1.8$ \\
\hline$p$-value & $<0.001$ & $<0.001$ & $<0.001$ & $<0.001$ \\
\hline
\end{tabular}

in the CYP4F2, APOE, CALU, GGCX and FVII genes and observed only a small increment [30,32,33,35].

The TTR, a tool of measuring the achievement of INR target values over time, has been used in the setting of clinical trials to evaluate the effectiveness of warfarin therapy, particularly when warfarin is being compared with some other strategy [46]. Also, there is evidence that better anticoagulation control (i.e., higher TTR) can protect patients from severe or even fatal adverse events $[46,47]$. Based on the median TTR over a one-year follow-up period as a cut-off, here used as a proxy of anticoagulation stability (TTR of 0.73 for patients in stable dose from the first cohort), we were able to find significant differences between the 'difference in weekly dose' for the PD and OD among algorithms (ours vs IWPC). That is, the closer an individual was, at the beginning of follow-up, to the predicted dose based on our algorithm (as opposed to a dose calculated by the IWPC algorithm), the more stable its anticoagulation regimen stayed during the next year. This suggests our algorithm to be not only associated with a more accurate dose determination, but also to be associated with a higher stability on anticoagulation therapy as compared with the IWPC algorithm in the Brazilian population.

Our study has some limitations. First, we included only adult individuals who had a target INR between 2 and 3 to model our algorithm. Thus, our results cannot be directly transposed to patients who have other target values or to children. Second, our study was a single center study; however, we were able to select - in different time periods - two large cohorts enrolling individuals from different ethnic and socio-economic backgrounds. Third, we were not able to consider dietary factors (especially vitamin $\mathrm{K}$ intake) and exercise level, but the impact of this variable is probably small [24,48-50].

Warfarin has been used worldwide for over 50 years and is effective to prevent and treat stroke and venous thromboembolism. Nonetheless, the challenges to personalize dosage are well-known. Optimization of the warfarin therapy with the use of tools like dosing-algorithms is being tested as a clinical strategy to improve effectiveness. However, the main randomized, controlled trials observed different findings in this regard. Of particular importance, these studies used different sampled populations and designs [12-14]. Consequently, clinical communities are undecided as to the usefulness of incorporating pharmacogenetic information into warfarin prescribing decisions. An often-cited reason is the paucity of randomized, controlled trials showing the superiority of genotype-informed dosing strategies over routine care. Our data suggest that it is important to develop population-specific algorithms to evaluate whether pharmacogenetic-based dosing can capture population-specific information and whether they are or not superior to local standards of care. This information should be taken into consideration in the design and interpretation of current clinical trials.

\section{Conclusion}

We were able to develop and validate our algorithm in two independent patient cohorts. Data suggest that our developed algorithm is more accurate than the IWPC algorithm when the application is focused on patients from the Brazilian population. These data suggest that population-specific derivation and/or calibration of warfarin dosing algorithms may lead to improved performance compared with general use currently available dosing algorithms.

\section{Future perspective}

Randomized studies using the developed algorithm could evaluate the impact of a population-specific genetic algorithm on the quality of the warfarin therapy and pharmacoeconomic data. 
Acknowledgements

The technical assistance of the Laboratory of Genetics and Molecular Cardiology group, Heart Institute (InCor) and Sociedade Hospital Samaritano - Ministério da Saúde (PROADI-SUS; SIPAR: 25000.180.672/2011-81) is gratefully acknowledged.

Financial \& competing interests disclosure

PCJL Santos and NE Duarte are recipients of fellowship from FAPESP, Brazil, Proc.2013/09295-3, Proc.2013/20614-3 and Proc. 2012/00316-5. The authors have no other relevant affiliations or financial involvement with any organization or entity with a financial interest in or financial conflict with the subject matter or materials discussed in the manuscript apart from those disclosed.
No writing assistance was utilized in the production of this manuscript.

\section{Ethical conduct of research}

The authors state that they have obtained appropriate institutional review board approval or have followed the principles outlined in the Declaration of Helsinki for all human or animal experimental investigations. In addition, for investigations involving human subjects, informed consent has been obtained from the participants involved.

\section{Open access}

This work is licensed under the Creative Commons AttributionNonCommercial 3.0 Unported License. To view a copy of this license, visit http://creativecommons.org/licenses/by-nc-nd/3.0/

Executive summary

\section{Background}

- Warfarin dose is associated with polymorphisms in the CYP2C9 and VKORC1 genes.

- The main aims of the present study were to develop a pharmacogenetic-based warfarin dosing algorithm and to validate it in a highly admixed population.

\section{Study design}

- We included two patient cohorts treated with warfarin.

- The algorithm was created using data from the first cohort of patients with warfarin stable dose.

- We used a second, independent, cohort to validate our algorithm.

Results

- The correlation between the predicted dose by our algorithm and the observed dose was higher than the correlation between the predicted dose by other tested algorithms and the observed dose.

- Data suggest that our developed algorithm is more accurate than the IWPC algorithm when the application is focused on patients from the Brazilian population.

\section{Conclusion}

- Population-specific derivation and/or calibration of warfarin dosing algorithms may lead to improved performance compared with general use dosing algorithms currently available.

\section{References}

1 Ageno W, Gallus AS, Wittkowsky A, Crowther M, Hylek EM, Palareti G. Oral anticoagulant therapy: antithrombotic therapy and prevention of thrombosis, 9th ed: American College of Chest Physicians EvidenceBased Clinical Practice Guidelines. Chest 141(2 Suppl.), e44S-S88 (2012).

2 Guyatt GH, Akl EA, Crowther M, Gutterman DD, Schuunemann HJ. Executive summary: antithrombotic therapy and prevention of thrombosis, 9th ed: American College of Chest Physicians Evidence-Based Clinical Practice Guidelines. Chest 141(2 Suppl.), S7-S47 (2012).

3 Bleeding during antithrombotic therapy in patients with atrial fibrillation. The stroke prevention in atrial fibrillation investigators. Arch. Intern. Med. 156(4), 409-416 (1996).

4 Adjusted-dose warfarin versus low-intensity, fixed-dose warfarin plus aspirin for high-risk patients with atrial fibrillation: stroke prevention in atrial fibrillation III randomised clinical trial. Lancet 348(9028), 633-638 (1996).
5 Bodin L, Verstuyft C, Tregouet DA et al. Cytochrome P450 2C9 (CYP2C9) and vitamin K epoxide reductase (VKORC1) genotypes as determinants of acenocoumarol sensitivity. Blood 106(1), 135-140 (2005).

6 Santos PC, Dinardo CL, Schettert IT et al. CYP2C9 and VKORC1 polymorphisms influence warfarin dose variability in patients on long-term anticoagulation. Eur. J. Clin. Pharmacol. 69(4), 789-797 (2013).

7 Sutfin T, Balmer K, Bostrom H, Eriksson S, Hoglund P, Paulsen O. Stereoselective interaction of omeprazole with warfarin in healthy men. Ther. Drug Monit. 11(2), 176-184 (1989).

8 Loebstein R, Dvoskin I, Halkin $\mathrm{H}$ et al. A coding VKORC1 Asp36Tyr polymorphism predisposes to warfarin resistance. Blood 109(6), 2477-2480 (2007).

9 Gage BF, Eby C, Milligan PE, Banet GA, Duncan JR, McLeod HL. Use of pharmacogenetics and clinical factors to predict the maintenance dose of warfarin. Thromb. Haemost. 91(1), 87-94 (2004).

10 Coumadin (warfarin sodium) (package insert). Bristol-Myers Squibb, Princeton, NJ, USA. www.accessdata.fda.gov 
11 Zineh I, Pacanowski M, Woodcock J. Pharmacogenetics and coumarin dosing - recalibrating expectations. N. Engl. J. Med. 369(24), 2273-2275 (2014).

12 Pirmohamed M, Burnside G, Eriksson N et al. A randomized trial of genotype-guided dosing of warfarin. N. Engl. J. Med. 369(24), 2294-2303 (2013).

13 Verhoef TI, Ragia G, de Boer A et al. A randomized trial of genotype-guided dosing of acenocoumarol and phenprocoumon. N. Engl. J. Med. 369(24), 2304-2312 (2013).

14 Kimmel SE, French B, Kasner SE et al. A pharmacogenetic versus a clinical algorithm for warfarin dosing. N. Engl. J. Med. 369(24), 2283-2293 (2013).

15 Soares RA, Santos PC, Machado-Coelho GL et al. CYP2C9 and $V K O R C 1$ polymorphisms are differently distributed in the Brazilian population according to self-declared ethnicity or genetic ancestry. Genet. Test. Mol. Biomarkers 16(8), 957-963 (2012).

16 Santos PC, Soares RA, Santos DB et al. CYP2C19 and $A B C B 1$ gene polymorphisms are differently distributed according to ethnicity in the Brazilian general population. BMC Med. Genet. 12, 13 (2011).

17 Pisters R, Lane DA, Nieuwlaat R, de Vos CB, Crijns HJ, Lip GY. A novel user-friendly score (HAS-BLED) to assess 1-year risk of major bleeding in patients with atrial fibrillation: the Euro Heart Survey. Chest 138(5), 1093-1100 (2010).

18 Ansell J, Hirsh J, Hylek E, Jacobson A, Crowther M, Palareti G. Pharmacology and management of the vitamin $\mathrm{K}$ antagonists: American College of Chest Physicians EvidenceBased Clinical Practice Guidelines (8th Edition). Chest 133(6 Suppl.), S160-S198 (2008).

19 Rosendaal FR, Cannegieter SC, van der Meer FJ, Briet E. A method to determine the optimal intensity of oral anticoagulant therapy. Thromb. Haemost. 69(3), 236-239 (1993).

20 Becker RC, Bassand JP, Budaj A et al. Bleeding complications with the P2Y12 receptor antagonists clopidogrel and ticagrelor in the PLATelet inhibition and patient Outcomes (PLATO) trial. Eur. Heart J. 32 (23), 2933-2944 (2011).

21 Jetter A, Kinzig-Schippers M, Skott A, Lazar A et al. Cytochrome P450 2C9 phenotyping using low-dose tolbutamide. Eur. J. Clin. Pharmacol. 60(3), 165-171 (2004).

22 Sconce EA, Khan TI, Wynne HA et al. The impact of CYP2C9 and VKORC1 genetic polymorphism and patient characteristics upon warfarin dose requirements: proposal for a new dosing regimen. Blood 106(7), 2329-2333 (2005).

23 Scott SA, Khasawneh R, Peter I, Kornreich R, Desnick RJ. Combined CYP2C9, VKORC1 and CYP4F2 frequencies among racial and ethnic groups. Pharmacogenomics 11(6), 781-791 (2010).

24 Klein TE, Altman RB, Eriksson N et al. Estimation of the warfarin dose with clinical and pharmacogenetic data. N. Engl. J. Med. 360 (8), 753-764 (2009).

25 Wadelius M, Chen LY, Lindh JD et al. The largest prospective warfarin-treated cohort supports genetic forecasting. Blood 113(4), 784-792 (2009).
26 Cho HJ, On YK, Bang OY et al. Development and comparison of a warfarin-dosing algorithm for Korean patients with atrial fibrillation. Clin. Ther. 33(10), 1371-1380 (2011).

27 Wu AH, Wang P, Smith A et al. Dosing algorithm for warfarin using CYP2C9 and VKORC1 genotyping from a multi-ethnic population: comparison with other equations. Pharmacogenomics 9(2), 169-178 (2008).

28 Shaw PB, Donovan JL, Tran MT, Lemon SC, Burgwinkle P, Gore J. Accuracy assessment of pharmacogenetically predictive warfarin dosing algorithms in patients of an academic medical center anticoagulation clinic. J. Thromb. Thrombolysis 30(2), 220-225 (2010).

Zhu Y, Shennan M, Reynolds KK et al. Estimation of warfarin maintenance dose based on VKORC1 (-1639 G>A) and CYP2C9 genotypes. Clin. Chem. 53(7), 1199-1205 (2007).

30 Botton MR, Bandinelli E, Rohde LE, Amon LC, Hutz MH. Influence of genetic, biological and pharmacological factors on warfarin dose in a Southern Brazilian population of European ancestry. Br. J. Clin. Pharmacol. 72(3), 442-450 (2011).

31 Perini JA, Struchiner CJ, Silva-Assuncao E et al. Pharmacogenetics of warfarin: development of a dosing algorithm for Brazilian patients. Clin. Pharmacol. Ther. 84(6), 722-728 (2008).

32 Krishna Kumar D, Shewade DG, Loriot MA et al. Effect of CYP2C9, VKORC1, CYP4F2 and GGCX genetic variants on warfarin maintenance dose and explicating a new pharmacogenetic algorithm in South Indian population. Eur. J. Clin. Pharmacol. 70(1), 47-56 (2014).

33 Mazzaccara C, Conti V, Liguori R et al. Warfarin anticoagulant therapy: a Southern Italy pharmacogeneticsbased dosing model. PLoS ONE 8(8), e71505 (2008).

34 Botton MR, Hutz MH, Suarez-Kurtz G. Influence of the CYP2C9*3 allele on the pharmacological interaction between warfarin and simvastatin. Pharmacogenomics 13(14), 1557-1559; author reply 1561-1552 (2013).

35 Ramos AS, Seip RL, Rivera-Miranda G et al. Development of a pharmacogenetic-guided warfarin dosing algorithm for Puerto Rican patients. Pharmacogenomics 13(16), 1937-1950 (2012).

36 Suarez-Kurtz G, Paula DP, Struchiner CJ. Pharmacogenomic implications of population admixture: Brazil as a model case. Pharmacogenomics 15(2), 209-219 (2014).

37 Parra FC, Amado RC, Lambertucci JR, Rocha J, Antunes CM, Pena SD. Color and genomic ancestry in Brazilians. Proc. Natl Acad. Sci. USA 100(1), 177-182 (2003).

Giolo SR, Soler JM, Greenway SC et al. Brazilian urban population genetic structure reveals a high degree of admixture. Eur. J. Hum. Genet. 20(1), 111-116 (2012).

39 Pena SD, Di Pietro G, Fuchshuber-Moraes M et al. The genomic ancestry of individuals from different geographical regions of Brazil is more uniform than expected. PLoS ONE 6(2), e17063 (2008).

40 Ozer N, Cam N, Tangurek B et al. The impact of CYP2C9 and VKORC1 genetic polymorphism and patient characteristics upon warfarin dose requirements in an adult Turkish population. Heart Vessels 25(2), 155-162 (2010). 
41 Miao L, Yang J, Huang C, Shen Z. Contribution of age, body weight, and CYP2C9 and VKORC1 genotype to the anticoagulant response to warfarin: proposal for a new dosing regimen in Chinese patients. Eur. J. Clin. Pharmacol. 63(12), 1135-1141 (2007).

42 Limdi NA, Beasley TM, Crowley MR et al. VKORC1 polymorphisms, haplotypes and haplotype groups on warfarin dose among African-Americans and EuropeanAmericans. Pharmacogenomics 9(10), 1445-1458 (2008).

43 Cavallari LH, Langaee TY, Momary KM et al. Genetic and clinical predictors of warfarin dose requirements in African Americans. Clin. Pharmacol. Ther. 87(4), 459-464 (2010).

44 Rieder MJ, Reiner AP, Gage BF et al. Effect of VKORC1 haplotypes on transcriptional regulation and warfarin dose. N. Engl. J. Med. 352(22), 2285-2293 (2005).

45 Wadelius M, Chen LY, Eriksson N et al. Association of warfarin dose with genes involved in its action and metabolism. Hum. Genet. 121(1), 23-34 (2007).

46 Rose AJ, Ozonoff A, Berlowitz DR, Henault LE, Hylek EM Warfarin dose management affects INR control. J. Thromb. Haemost. 7(1), 94-101 (2009).
47 Connolly SJ, Pogue J, Eikelboom J et al. Benefit of oral anticoagulant over antiplatelet therapy in atrial fibrillation depends on the quality of international normalized ratio control achieved by centers and countries as measured by time in therapeutic range. Circulation 118(20), 2029-2037 (2008).

48 Wei M, Ye F, Xie D et al. A new algorithm to predict warfarin dose from polymorphisms of CYP4F2, CYP2C9 and VKORC1 and clinical variables: derivation in Han Chinese patients with non valvular atrial fibrillation. Thromb. Haemost. 107(6), 1083-1091 (2012).

49 Franco V, Polanczyk CA, Clausell N, Rohde LE. Role of dietary vitamin $\mathrm{K}$ intake in chronic oral anticoagulation: prospective evidence from observational and randomized protocols. Am. J. Med. 116(10), 651-656 (2004).

50 You JH, Wong RS, Waye MM et al. Warfarin dosing algorithm using clinical, demographic and pharmacogenetic data from Chinese patients. J. Thromb. Thrombolysis 31(1), 113-118 (2011). 